\title{
LOS DERECHOS DE PROPIEDAD: UN ELEMENTO CENTRAL PARA EL DESARROLLO ECONÓMICO
}

\author{
Yamile Rivera Romero ${ }^{1}$
}

Recibido: 16 de mayo de 2013

Aceptado: 23 de agosto de 2013

\section{Resumen}

El propósito de este artículo es analizar el impacto de los derechos de propiedad sobre el desarrollo económico. Para este efecto, se tomó como base los planteamientos de la teoría neoinstitucional sobre las instituciones y su impacto sobre el comportamiento humano. Definición a partir de la cual se establece si los derechos de propiedad pueden ser considerados como una institución, que ejerce influencia en los incentivos de los agentes económicos y de esta forma en los resultados económicos de una nación. Finalmente, se concluye que la conducta de los agentes y el uso dado por éstos a los recursos económicos no solo están afectados por los movimientos de los precios propios de los mercados de bienes y factores, sino por la estructura de derechos de propiedad vigente en el marco institucional bajo el cual se producen las relaciones productivas. Por tanto, se reconoce que los derechos de propiedad generan los incentivos para la maximización de resultados y en general, para un mejor desempeño económico..

Palabras claves: Propiedad, instituciones, derechos de propiedad, desarrollo económico, neo-institucionalismo.

\section{THE PROPERTY RIGTHS: AN CENTRAL ELEMENT TO ECONOMIC DEVELOPMENT}

\section{Abstract}

The aim of this paper is to analyze the role of property rights on economic development. This began theoretical construction of neo-institutionalism theories about institutions and their impact on human behavior. This definition lets set if the property rights can be considered as an institution, which exerts influence on the incentives of economic agents, and thus in the economic results of a nation. Finally, it is concluded that the conduct of agents and how they use the economic resources not only are affected by movements of goods and factors' prices, but by the structure of existing property rights within the institutional framework under which the productive relations occur. By this way, it recognizes the rights of property to generate incentives for the maximization of results and in general, for better economic performance.

Keywords: Property, institutions, property rights, economic development, neo-institutionalism.

\footnotetext{
${ }^{1}$ Magíster en Ciencias Económicas, Especialista en Finanzas y Economista. Docente de la Universidad 


\section{Introducción}

La línea de investigación propuesta por el neoinstitucionalismo destaca la existencia de dos elementos estrechamente vinculados entre sí y que resultan determinantes para el desarrollo económico: el Estado y el sistema de derechos de propiedad. Puesto que, «sí no existiera el estado, ni sus instituciones, ni tampoco un sistema de derechos de propiedad en el que éstos puedan apoyarse, surgirían costos de transacción muy elevados que paralizarían los sistemas de producción [...] y no [se emprenderían las inversiones requeridas para la ocurrencia de] relaciones de intercambio de largo plazo» (Eggertsson, 1995).

Aunque, el reconocimiento que las instituciones afectan los resultados económicos pueden ser trazado de regreso a Adam Smith (1776), hasta llegar al trabajo de Douglass North (1973), «fue Marx el primero en afirmar abiertamente, que la especificación de los derechos de propiedad podría dar respuesta a las cuestiones sociales derivados del problema de la escasez y que las estructuras de derechos de propiedad [afectan] el comportamiento económico de manera específica y predecible» (Eggertsson, 1995).

En particular, la historia de las democracias occidentales resalta el papel clave que desempeñan los derechos de propiedad en la promoción del desarrollo económico y político. La forma en que estos países han definido y protegido los derechos de propiedad a través del tiempo puede constituir una valiosa guía para otras naciones que están buscando el establecimiento de instituciones legales, con el propósito de crear sistemas vigorosos de propiedad privada.

Dado que la distribución de los derechos de propiedad ejerce influencia en los incentivos y en el comportamiento humano, y de esta forma en los resultados económicos de una nación; existe una base para estudiar el impacto de los acuerdos sobre derechos de propiedad en los resultados económicos. Por tanto, el presente artículo tiene por objeto reconocer el papel que desempeñan las estructuras de derechos de propiedad en el desarrollo económico.

\section{Derechos de propiedad: una aproximación conceptual}

La propiedad no es algo que se posee - es un conjunto de derechos. Tales derechos históricamente han estado clasificados en tres categorías. «En primer lugar, existen derechos sobre la utilización de un activo - derechos de usuario - que definen todos los usos posibles que legalmente tiene el individuo sobre un activo, incluyendo el derecho a su transformación física o incluso a su destrucción. En segundo lugar, existe el derecho a obtener una renta de un activo y a pactar los términos de un contrato con otros individuos. [Y por último], existe el derecho a transferir permanentemente los derechos de propiedad sobre un activo a otro individuoes decir, [a realizar su enajenación o venta]" (Eggertsson, 1995).

Por consiguiente, los derechos de propiedad sobre un bien son considerados como un conjunto de facultades legales que pueden intercambiarse libremente y de las cuales depende la asignación y la transformación productiva de los recursos. Así, un sistema de derechos de propiedad puede ser concebido como «un método mediante el cual se confiere a los individuos la potestad de seleccionar, para bienes específicos, cualquier uso entre todo un conjunto de usos posibles no prohibidos" (Eggertsson, 1995).

Adicionalmente, es importante notar que el contenido de los derechos otorgados está determinado por el marco económico y político vigente o por la forma en que se regulan los derechos de propiedad. Este hecho está relacionado con los modos particulares adoptados por una sociedad para resolver los problemas asociados 
con la escasez de recursos (Alchian y Demsetz, 1973). Así, por ejemplo, el capitalismo descansa en los mercados y en los derechos de propiedad privada para resolver los conflictos sobre el uso de los recursos escasos.

Estados Unidos es un destacado ejemplo de ello. En los años de formación del país se otorgaba un lugar prominente a la protección de la propiedad privada. La filosofía sobre la propiedad que apuntalaba el sistema norteamericano se basaba principalmente en la importancia de las libertades individuales, en las limitaciones del poder gubernamental y en el derecho de las personas a la propiedad. Este amplio concepto de la propiedad trascendía la posesión tangible de la propiedad privada, incluyendo la libertad y marcaba los límites de la intervención gubernamental sobre sus ciudadanos.

En contraste, en América Latina se favorecieron estructuras de concentración de la propiedad, lo cual se profundizó por la presencia de un Estado poderoso para confiscar la riqueza de los ciudadanos. Por tanto, no se promovió el respeto a la propiedad individual, sino que se permitió la aplicación de políticas sobre la tierra, el capital, la información, etc., que parecen haber aunado la desigualdad en las relaciones de propiedad.

\section{Los derechos de propiedad y el desarrollo económico}

La economía neo-institucional reconoce que «las elecciones económicas individuales no responden exclusivamente a cambios en los precios relativos que ocurren en el mercado de productos y factores, sino también a los estímulos provenientes del entorno institucional, como la información disponible o la definición de los derechos de propiedad» (Ayala, 1999). Por tanto, «la estructura institucional provee los incentivos que dictan las clases de habilidades y conocimientos percibidos para la maximización de resultados. [Así] las instituciones en las sociedades [...] son el determinante subyacente del desempeño de las economías» (North, 1993).

De conformidad con lo anterior, es claro que una modificación de la definición de los derechos de propiedad altera significativamente la conducta de los agentes, puesto que los individuos al tomar sus decisiones lo hacen basados en la posición que ocupan en las relaciones de propiedad vigentes, esto es, considerando sobre cuales bienes pueden ejercer propiedad. En este sentido, se advierte que "cuando existe el acceso y los derechos a usar la propiedad, los agentes no buscan maximizar sólo en lo inmediato y en el corto plazo, sino que toman en consideración los costos y beneficios en el largo plazo» (Ayala, 1999). De esta forma, unos derechos de propiedad seguros generan los estímulos necesarios para la innovación, limitando el uso predatorio de los bienes.

Luego, ¿Por qué son tan importantes los derechos de propiedad en el desarrollo económico? Al respecto, «North anota que Adam Smith consideraba que los incentivos individuales que conducían a la mayor riqueza podían arrastrar a toda la sociedad en la misma dirección, pero con la condición de que existieran instituciones que condujeran a este resultado. (...) Los dos movimientos, individual y social, podían divergir sí los derechos de propiedad estaban especificados en forma incompleta o eran aplicados en forma inconsistente. En tales casos, habría efectos de un tercer agente, de tal modo que parte de las ganancias y pérdidas serían capturadas por fuera del intercambio entre los agentes» (Kalmanovitz, 2001).

Este planteamiento es desarrollado por North y Thomas (1973), quienes con base en la teoría de los derechos de propiedad, explican el desarrollo económico de Europa occidental desde la Edad Media hasta el siglo XVIII. La tesis central de North y Thomas (1973), es que el desarrollo de una organización económica eficiente fue fundamental para el crecimiento económico 
per cápita de Europa occidental. Argumentan que una organización eficiente conduce al establecimiento de arreglos institucionales y de una estructura de derechos de propiedad que crean incentivos para canalizar el esfuerzo económico individual hacia actividades productivas. Dicho de otro modo, tal organización contribuye a aumentar la tasa privada de retorno de los emprendimientos productivos $\mathrm{y}$, por consiguiente, a estimular el desarrollo económico.

Sin embargo, cuando los derechos de propiedad no están bien definidos o no se los hace cumplir, se genera una discrepancia entre los beneficios privados y sociales o los costos ${ }^{2}$. Tal equiparación de ganancias, en particular, no se ha producido al menos por dos razones, relacionadas con la evolución histórica de los derechos de propiedad: i) «Es posible que no exista la técnica adecuada para hacer frente al free rider y forzar a todos los implicados a hacerse cargo de su parte en los costos de transacción. (...) En diversas épocas se han ideado sistemas - secretos, recompensas, premios, derechos de autor, patentes - para conseguir la equiparación entre beneficios privados y sociales; pero incluso en nuestros días las técnicas para mantener a los intrusos al margen de los beneficios continúan siendo costosas e imperfectas. $Y$ ii) para un grupo o un individuo, los costos de crear o hacer cumplir los derechos de propiedad pueden superar a los beneficios» (North y Thomas, 1973).

La situación anterior, significa, «que terceras personas, sin su consentimiento, recibirán algunos de los beneficios o incurrirán en algunos de los costos. [Por tanto], si los costos privados exceden a los beneficios privados, los individuos

\footnotetext{
Un ejemplo de la historia, que ofrece North y Thomas (1973), «procede de la política respecto a la tierra que se puso en práctica en España a comienzos de la Edad Moderna. Conforme la tierra se hizo más escasa debido al crecimiento de la población, aumentó la tasa social de beneficios derivada del aumento de la eficacia de la agricultura, pero no así la privada, porque la Corona había concedido previamente a la asociación de ganaderos (la Mesta) derechos exclusivos para conducir sus rebaños a través de España en su manera acostumbrada. Un propietario que preparase y cultivase cuidadosamente su cosecha podía ver en cualquier momento cómo los rebaños de ovejas de la comín o la pisoteaban. En este caso el dueño nominal no contaba con un derecho exclusivo sobre sus tierras".
}

no estarán dispuestos a emprender esa actividad, aun cuando ésta sea socialmente lucrativa» (North y Thomas, 1973).

Luego, la amplitud de la brecha entre la tasa social y la tasa privada de retorno es inversamente proporcional a la eficiencia del marco institucional. Así, la innovación tecnológica, las economías de escala, la mejora de la educación y la acumulación de capital, como parte del desarrollo económico; no ocurrirán a menos que la organización económica existente sea eficiente.

Se sigue, por tanto, que es esencial contar con una teoría de los derechos de propiedad $^{3}$ que, por un lado, sea capaz de describir los incentivos económicos individuales y grupales con que cuenta el sistema y, por el otro, pueda explicar las formas de organización económica que los seres humanos diseñan para reducir costos de transacción y organizar el intercambio. De esta forma, la propiedad es una institución socialmente deseable e individualmente rentable, cuya existencia depende de la capacidad tecnológica y organizativa de la sociedad para establecer los sistemas para su protección. No obstante, se señala que la creación, especificación y aplicación de los derechos de propiedad son costosas, de tal suerte que si los costos superan a los beneficios, el mismo mercado desplaza a la institución.

En este marco, surge un dilema político fundamental para el sistema económico, esto es, las tensiones nacidas de la necesidad de crear un gobierno suficientemente fuerte para proteger los derechos de propiedad (North y Weingast, 1989). La respuesta a una situación como ésta, es el diseño de instituciones políticas que comprometan de manera creíble al Estado en preservar los mercados, es decir, en limitar hacia el futuro la discreción política respecto a la economía, puesto que de esta forma permite que

\footnotetext{
La teoría de las instituciones propuesta por North tiene tres componentes básicos: a) una teoría de los derechos de propiedad; b) una teoría del Estado y c) una teoría de la ideología (North, 1981)
} 
los derechos privados y el intercambio prevalezcan en grandes segmentos de la economía. En contraste, la ausencia de un compromiso creíble con las políticas y reglas que sean coherentes con la economía de mercado y respeten la propiedad, significa la emisión de señales negativas para los actores privados y finalmente, conlleva la detención de la actividad económica necesaria para el desarrollo económico.

Los gobiernos, para North y Thomas (1973), asumen la protección y aplicación de los derechos de propiedad porque ellos pueden hacerlo a un menor costo frente a los grupos privados, que podrían organizarse voluntariamente. Sin embargo, los gobiernos apremiados por sus necesidades fiscales, pueden establecer y proteger ciertos derechos de propiedad que obstaculicen el desarrollo en vez de alentarlo, no hay garantías de que los arreglos institucionales requeridos sean los que se instauren.

Con este argumento parece confirmarse una de las objeciones al viejo liberalismo. Este suponía que en el estado natural ya existían amplios derechos de propiedad y que la tarea del gobierno consistía en proteger esos derechos. Pero, esto es objetable porque depende de unas premisas dudosas: que los derechos de propiedad existen de forma independiente antes de la existencia del Estado y porque impone al gobierno la obligación de conservar y reforzar las desigualdades heredadas. Parece, por tanto, más adecuado, siguiendo la hipótesis de North (1993), afirmar que el nacimiento del Estado y sus instituciones legales fueron las que facilitaron e hicieron que fuera individualmente rentable la propagación de la propiedad.

Por otra parte, Coase (1960) entrega una aproximación útil del papel de los determinantes de los derechos de propiedad. El autor muestra, que un mundo hipotético en el que los costos de asegurar los derechos de propiedad y de medir, monitorear y transferir los bienes son bajos, la distribución inicial de los recursos y derechos no tendrá efectos sobre la asignación eficiente de los bienes, puesto que los derechos de propiedad siempre se dirigirán a su uso más valioso. Sin embargo, ese mundo no corresponde al nuestro.

Para Coase (1988), es claro que el mundo actual no es un escenario con cero costos de transacción, medición, monitoreo, aseguramiento de la propiedad, intercambio y obtención de la información. En el mundo real los costos de transacción determinan los derechos de propiedad, la extensión del intercambio, la especialización y la productividad. Sí los costos de transacción disminuyen, los derechos de propiedad serán más claramente definidos, más bienes y servicios serán intercambiados, los beneficios de la especialización se incrementarán y mayores ganancias económicas serán realizadas. Esto es así, porque se espera que, cuando los costes de transacción sean elevados los gobernantes por su propio interés, diseñen estructuras adecuadas de derechos de propiedad para que la economía crezca rápidamente.

Por tanto, en el mundo real de costos de transacción positivos, las dificultades para la consecución del desarrollo son más complicadas y especialmente, si se admite que deben producirse inevitablemente ciertas fricciones entre la creación de una serie de derechos de propiedad y el funcionamiento del sistema una vez que dichos derechos se han incorporado a él de un modo efectivo. Además, «los derechos de propiedad, lejos de ser (...) flexibles, [en ocasiones] se sitúan a la zaga de los cambios que experimenta el entorno y actúan como freno del desarrollo y del crecimiento económico» (Eggertsson, 1995) ${ }^{4}$, debido a que toda modificación de

"Los sistemas políticos y económicos de éxito han desarrollado estructuras institu-
cionales flexibles que pueden sobrevivir a las sacudidas y cambios que son parte del
desarrollo próspero. Pero estos sistemas han sido producto de una larga gestación» (North, 1994) 
los mismos altera los incentivos de los agentes y por esta vía, la asignación productiva de los recursos, la composición de los bienes producidos y la distribución de los ingresos. Luego, la emergencia de instituciones políticas que especifiquen derechos de propiedad eficientes y provean una efectiva ejecución de los mismos, es un elemento medular para el alcance del desarrollo económico.

Así, la existencia en un sistema económico de una estructura de derechos de propiedad sólida y creíble es clave en la interacción social, pues reduce la incertidumbre del agente, especifica los beneficios que puede obtener de la cooperación y determina así sus incentivos, puesto que define la posición del individuo con respecto a la utilización de los recursos escasos (Kalmanovitz, 2001). En este sentido, «la definición e interpretación de los derechos de propiedad por medio de las cortes, legislaturas, burocracias, etc., afecta las elecciones de los agentes porque la seguridad sobre el respeto a los derechos de propiedad puede significar que los individuos se involucren en la organización de negocios complejos y de larga maduración» (Ayala, 1999).

Por consiguiente, «la disponibilidad o inexistencia de información acerca de los derechos de propiedad afecta sensiblemente la toma de decisiones, [puesto que] ninguna inversión importante se realizará si no existe información cierta sobre los alcances y límites de los derechos de propiedad» (Ayala, 1999). De esta forma, los derechos de propiedad, actúan como mecanismo institucional de coordinación en el costoso proceso del intercambio, ya que contribuyen al mejor entendimiento del proceso de asignación de recursos. En suma, los derechos de propiedad son un importante elemento de coordinación entre agentes dado que representan un elemento institucional relevante en lo que se refiere a la asignación y uso de los recursos disponibles y por ende, para el intercambio.
En consecuencia, para que la actividad económica tenga lugar, los derechos de propiedad deben estar claramente definidos y los resultados de las acciones legales deben ser fáciles de predecir. Esto es cierto, sí se considera que al mejorar la claridad de la relación de propiedad se estimula a los propietarios actuales y potenciales a buscar los usos más valiosos para los recursos, a través de un amplio conjunto de individuos y sobre un horizonte más largo de tiempo. Por ejemplo, permite que los propietarios de activos accedan a los mercados de capital usando estos activos como colateral.

Además, el sistema de derechos de propiedad, debe ser ideado de tal forma que permita a los individuos apropiarse de los beneficios derivados de las actividades productivas adelantadas, pero al mismo tiempo, haciéndoles responsables por los costos impuestos a los demás y facilitando la transferencia de derechos entre individuos (Kalmanovitz, 2001). El conjunto de incentivos, generado por este, debe premiar con mayores ganancias la creación de procesos que abaraten las mercancías y los insumos, que innoven en los productos, que mejoren su calidad y que se puedan intercambiar sus derechos libremente, en la forma de suscribir acciones nuevas, hacer alianzas estratégicas, vender el capital, etc. Los derechos de propiedad deben ser generales y no tener excepciones. De igual manera, las responsabilidades por las acciones de los agentes deben ser cobradas sin excepción.

\section{Resultados Empíricos}

Zak (2001) estableció que existe un vínculo entre instituciones, derechos de propiedad y crecimiento. Para este efecto, este autor modela una situación en la que los derechos de propiedad son inseguros $y$, además, se incurre en altos costos para su cumplimiento.

En particular, el modelo establece que las economías en las cuales la estructura institucional 
no protege adecuadamente los derechos de propiedad crecen lentamente, o no lo hacen, en tanto que los países con una mejor protección crecen de conformidad con el modelo neoclásico estándar.

Según el autor, la amenaza de pérdida de la propiedad genera los incentivos para establecer las instituciones que garanticen los derechos de propiedad. Debido a que los propietarios de recursos tienen interés en la protección de los derechos de propiedad, ellos son solamente un grupo de agentes que estarían dispuestos a pagar por la formación de instituciones para reforzar los derechos de propiedad. Por tanto, se examina el papel que una institución, la cual se denomina gobierno, tiene sobre la trayectoria de crecimiento de la economía.

Los tests empíricos ${ }^{5}$ de las predicciones del modelo demuestran que los gastos del gobierno dirigidos a garantizar los derechos de propiedad incrementan el crecimiento per cápita del ingreso. Así mismo, éste advierte que los países en desarrollo, en los que los derechos de propiedad son inseguros, corren el riesgo de tender hacia el empobrecimiento, igualmente sí la política del gobierno para garantizar los derechos de propiedad es óptima. En este contexto, Zak (1991) señala que existe una relación positiva entre la seguridad en los derechos de propiedad y el ingreso de largo plazo

En síntesis, el artículo apoyado en un modelo de crecimiento, en el cual los derechos de propiedad son inseguros y costosos para reforzar, afirma que la garantía imperfecta de los derechos de propiedad posiblemente explique parte de las fallas del crecimiento. Así mismo, indica que la posible solución es la formación de instituciones que garanticen el respeto a la propiedad.

Estimación de las funciones de política óptima del gobierno para la protección de los derechos y ecuaciones de crecimiento de un conjunto amplio de países (Zak, 1991).

En las sociedades democráticas, los cambios fundamentales de derechos de propiedad se producen a través de algún tipo de procedimiento electoral, bien sea de forma directa por medio del voto popular o de forma indirecta a través del voto de representantes electos (Eggertsson, 1995)

\section{Conclusiones}

La asignación de recursos en las sociedades modernas está condicionada por los cambios en la estructura de las reglas, esto es por la reasignación de los derechos de propiedad ${ }^{6}$ y en cierto grado, por los cambios en las instituciones sociales y políticas. Por tanto, la clarificación de los derechos de propiedad, la ejecutabilidad de los contratos y la predictibilidad y estabilidad de las reglas de juego en conjunto ofrecen un marco de incentivos para propiciador la actividad económica privada en el mercado. Esto, es clave sí se considera que las inversiones a largo plazo tienden a ser vulnerables a la apropiación indebida y, por tanto, únicamente tienen salida cuando los inversores potenciales pueden cerciorase de que los derechos de propiedad presentes, así como sus expectativas futuras, están suficientemente garantizados

Finalmente, debe señalarse que las instituciones formales particularmente influyentes en el desarrollo económico están representadas por los niveles de regulación de los derechos de propiedad, la ejecución de los contratos y la garantía de la ley. Por tanto, parte fundamental de una política de desarrollo es el diseño de un marco institucional que a su vez establezca y haga cumplir los derechos de propiedad eficientes, generando los incentivos para la maximización de resultados y en general, para un mejor desempeño económico.

\section{Referencias}

Alchian, A. y Demsetz, H. (1973). The property rights paradigm. Journal of Economic History, No 33, marzo.

Ayala, J. (1999). Instituciones y economía: Una introducción al Neo-institucionalismo económico. México: Fondo De Cultura Económica,

Barzel, Y. (1989). Economic analysis of property rights. Cambridge: Cambridge University Press.

Benham, A. y Benham, L. (1998). Property rights in transition economies: a commentary on what economists know. En Transforming Post-Communist Political Economies, Washington, D.C.: National Academy Press. 
Eggertsson, T. (1995). El comportamiento económico y las instituciones. Madrid: Editorial Alianza Economía.

Furubont, E. y Ritchter, R. (1997). Institutions and economic theory. Ann Arbor: Michigan University Press.

Hodgson, G. (1989). Institutional rigidities and economic growth. Cambridge Journal of Economics, No 13.

Kalmanovitz, S. (2001). Las Instituciones y desarrollo económico en Colombia. Cali: Editorial Norma.

North, D. (1981). Estructura y cambio en la historia económica. Barcelona: Alianza Editorial.

North, D. (1993). Instituciones, cambio institucional y desempeño económico. México: Fondo de Cultura Económica.

North, D. (1994). Economic performance through time. American Economic Review, No 84.
North, D. y Thomas, P. (1973). The rise of the western world: A New Economic History. Cambridge: Cambridge University Press.

North, D. y Weingast, B. (1989). Constitutions and commitment: the evolution of institutions governing public choice in seventeenth-century England. The Journal of Economic History, Vol. 49, No 4.

Reynolds, L. (1983). The spread of economic growth to the third world. Journal of Economic Literature, No 21.

World Intellectual Property Organization (2009). The economics of intellectual property: Suggestions for further research in developing countries and countries with economies in transition, WIPO Publication No 1012. Recuperado el 25 de agosto de 2013 en el sitio Web: http:// www.wipo.int/ip-development/en/economics/

Zak, P. (2001). Institutions, property rights, and growth. The Gruter Institute Working Papers on Law, Economics, and Evolutionary Biology, Vol. 2, Issue 1, Article No 2. 